\title{
Skiba points for small discount rates
}

\author{
F.O.O. Wagener *
}

7th July 2004

\begin{abstract}
The present article uses perturbation techniques to approximate the value function of a economic minimisation problem for small values of the discount rate. This can be used to obtain the approximate location of Skiba states (or indifference thresholds) in the problem; these are states for which there are two distinct optimal state trajectories, converging to different optimal steady states. It is shown that the sets of indifference thresholds are locally smooth manifolds. For a simple example, all relevant quantities are computed explicitely. Moreover, the approximation can be used to obtain parameter-dependent approximations to indifference manifolds.
\end{abstract}

Keywords: Multiple optimal equilibria, indifference points, perturbation methods

\section{INTRODUCTION}

In economic theory there has been for a long time a preference, sometimes dogmatic, for results that ensured the existence of a unique and stable equilibrium of some kind in a system. For in that situation the long-run behaviour of optimising economic agents is - at least theoretically - determined and independent of the specific initial state of the system, and therefore presumably quite robust: minor model misspecifications or parameter uncertainties usually do not disturb the qualitative picture. In the context of optimal control theory, 'global asymptotic stability' results have been obtained in the 1970's by a number of people: see chapter 12 of Brock and Malliaris [2] for extensive references. By placing certain convexity assumptions on (the level sets of) the Hamilton function of the problem,

\footnotetext{
*Florian Wagener, CeNDEF, Universiteit van Amsterdam, Roetersstraat 11, 1018 WB Amsterdam, The Netherlands,f.o.o.wagener@uva.nl
} 
it is ensured that optimal solutions exist for all possible initial states, and that all optimal solutions tend to the same optimal steady state as time tends to infinity.

Skiba [16] and Dechert and Nishimura [6] seem to have been the first to give examples of economic optimal control problems for which there are several locally optimal steady states. Necessarily, there are indifference thresholds (usually called 'Skiba points') in these systems; an indifference threshold is an initial state at which two optimal solutions originate, each of them tending to a different optimal steady state.

The existence of such a threshold has several theoretical consequences: first, the behaviour of the optimising agent is not determined at an indifference threshold. Next, model misspecifications and parameter uncertainties can have pronounced effects in this context, possibly directing the agent to the 'wrong' steady state. It is this feature that motivates interest in the occurence of thresholds in studies of the economics of ecological systems, like lakes, forests, coral reefs, or the climate as a whole. If a model comparing costs and benefits of such a natural resource convinces policy makers that it is not worth the cost to keep this resource intact, they may opt for a policy that overuses or destroys it; but a small difference in the model specification might point to a policy that keeps the resource intact. There is a quickly growing literature on these kind of economic-ecological models, see for instance Brock and Starrett [3], Dechert and Brock [5], Mäler, Xepapadeas and de Zeeuw [13], and the paper [17] of the present author. There are many other examples of the occurrence of Skiba points; a good overview is given in the recent survey [7].

Indifference thresholds do not occur in linear-quadratic control problems, and their existence is therefore a typically nonlinear property of an economic problem, where a functional of the form

$$
J[x]=\int_{0}^{\infty} g(x, \dot{x}) \mathrm{e}^{-\rho t} \mathrm{~d} t
$$

has to be optimised. What is more, it is a global property, since nonlocal information about the problem is necessary to determine the position of indifference states. In general, numerical methods have to be employed to obtain this kind of information.

The present article's main thrust is that analytic or semi-analytic information about indifference states can be obtained if the discount rate $\rho$ is small, by viewing the reduced Hamilton equations, which are associated to the optimality problem, as non-conservative perturbations of conservative (Hamiltonian) equations. Using standard techniques from perturbation theory the approximate location of an indifference state is then related to the value of certain integrals; in some cases these integrals may be evaluated analytically an example is given in the last section - but usually numerical methods will have to be invoked. The philosophy of this approach, using computational methods to obtain information which is not within reach of analytic methods, is in the spirit of computational economics as for instance championed by Judd [12].

Parametrised families of control problems are then considered, whose optimal solutions all tend to steady states. If the discount rate is positive, generically there is an open set of parameter values, the indifference set, for which there are indifference thresholds and by 
consequence multiple optimal steady states in the problem. If the parameter takes a value in the boundary of this set, there is a heteroclinic connection between saddle points of the reduced Hamilton equations (cf. [17, 18]). The location of these bifurcation points can also be determined approximatively for small discount rates. As an application, a 'threshold bifurcation diagram' is computed for the example.

The article is organised as follows. In section 2, the necessary concepts from the theory of dynamic optimalisation problems are recalled, and the specific situation to be investigated in the rest of the article is described carefully: there are assumed to be two saddle equilibria in the phase space, which may or may not correspond to local or global equilibria in the state space. The first main result (theorem 1) of the article states that if there are two locally optimal equilibria, the indifference thresholds between these form (locally) smooth manifolds in state space. This clarifies a point which seems not to have been answered before in the literature, though there are already several numerical computations of indifference manifolds, see e.g. $[9,10]$. Though the proof confines itself to the restricted setting of two locally optimal equilibria, it is general and easily extendable to more complicated situations.

Section 3 derives a perturbation result, by which for small discount rates the value function is expressed in terms of properties of the system without discounting. Especially in systems with one-dimensional state spaces, this leads to analytic expressions for the value function in terms of certain explicit integrals. Applications of the result to problems with higher dimensional state spaces are sketched. Finally, section 4 applies the perturbation result to an example problem.

\section{SETUP}

2.1 The minimisation problem. In this subsection and the next, the optimalisation problem to be considered in the present article is introduced, and several general notions from the calculus of variations are recalled briefly. The restriction to the context of the calculus of variations is made to conveniently avoid some technicalities; the results obtained rest on general properties of the (reduced) Hamilton equations.

The state space $\mathcal{X}$ of the optimalisation problems considered in this article is always an open connected domain of $\mathbb{R}^{n}$. Let $g: \mathcal{X} \times \mathbb{R}^{n} \rightarrow \mathbb{R}$ be a smooth function that is strictly convex in the second variable; smooth will always mean 'infinitely often differentiable' in the following. This is not a great restriction: the function $g$ can be seen as an approximation to the 'data' of the problem; for convenience we can choose this approximation to be smooth.

Recall that a function is absolutely continuous if its derivative is locally Lebesgueintegrable (see for instance [15]). This class is more general than the class of continuous and piecewise smooth functions, but the reader can equally well take the latter class in mind. Denote by $\mathcal{D}_{\xi}$ the set of absolutely continuous functions $x:[0, \infty) \rightarrow X$ satisfying $x(0)=\xi$; these functions will usually be termed 'state trajectories' in the following. 
Let a functional $J: \mathcal{D}_{\xi} \rightarrow \mathbb{R}$ be given by

$$
J[x]=\int_{0}^{\infty} g(x, \dot{x}) \mathrm{e}^{-\rho t} \mathrm{~d} t,
$$

if the integral exists. The value function $V: \mathcal{X} \rightarrow \mathbb{R} \cup\{-\infty\}$ is defined as

$$
V(\xi)=\inf _{x \in \mathcal{D}_{\xi}} J[x] .
$$

The value function is continuous. If for $x \in \mathcal{D}_{\xi}$ the equality $V(\xi)=J[x]$ holds, then $x$ is called a minimiser of $J$ in $\mathcal{D}_{\xi}$.

2.2 Canonical equations. Unless stated differently, in the following the notation $f_{x}$ will always denote partial differentiation of the function $f$ with respect to the variable $x$. Given a state trajectory $x \in \mathcal{D}_{\xi}$, introduce the costate trajectory $y:[0, \infty) \rightarrow \mathbb{R}^{n}$ by setting

$$
y(t)=g_{\dot{x}}(x(t), \dot{x}(t)) \mathrm{e}^{-\rho t},
$$

for all $t \in[0, \infty)$. As $g$ is strictly convex in $\dot{x}$, this equation can be inverted to yield $\dot{x}=$ $\Psi(t, x, y)=\psi\left(x, y \mathrm{e}^{\rho t}\right)$. The pair $(x, y)$ is called the phase trajectory of $x$; it takes values in the phase space $X \times \mathbb{R}^{n}$. In the following, let $\pi$ denote the standard projection $\pi(x, y)=y$ from phase space to state space.

The Hamilton function $\mathcal{H}$ of the minimisation problem is given as ${ }^{1}$

$$
\begin{aligned}
\mathcal{H}(t, x, y) & =y \Psi(t, x, y)-g(x, \Psi(t, x, y)) \mathrm{e}^{-\rho t} \\
& =y \psi\left(x, y \mathrm{e}^{\rho t}\right)-g\left(x, \psi\left(x, y \mathrm{e}^{\rho t}\right)\right) \mathrm{e}^{-\rho t} .
\end{aligned}
$$

By differentiating this expression with respect to $y$, it follows that $\mathcal{H}_{y}=\Psi$ and $\mathcal{H}_{y y}=\Psi_{y}$. Taking derivatives with respect to $y$ on both sides of $y=g_{\dot{x}}(x, \Psi) \mathrm{e}^{-\rho t}$ yields moreover that

$$
I=\mathrm{e}^{-\rho t} g_{\dot{x} \dot{x}} \Psi_{y}=\mathrm{e}^{-\rho t} g_{\dot{x} \dot{x}} \mathcal{H}_{y y},
$$

where $I$ denotes the identity matrix. Since $g$ is assumed to be convex in $\dot{x}$, the matrix $g_{\dot{x} \dot{x}}$ is positive definite: therefore its inverse $\mathrm{e}^{-\rho t} \mathcal{H}_{y y}$ is positive definite as well, and $\mathcal{H}$ is strictly convex in $y$.

If $x \in \mathcal{D}_{\xi}$ is a minimiser, then the phase trajectory of $x$ is necessarily a solution to the canonical (or Hamilton) equations

$$
\dot{x}=\mathcal{H}_{y}, \quad \dot{y}=-\mathcal{H}_{x} .
$$

\footnotetext{
${ }^{1}$ We conform to the classical sign convention $\mathcal{H}=y \dot{x}-g$ instead of the equivalent $\mathcal{H}=y \dot{x}+g$ in the tradition of Pontryagin and his coworkers [14], who formulated the necessary conditions for obtaining a minimum as a maximum principle.
} 
Generally, solution curves of these equations, even if they do not correspond to minimisers, will be termed phase curves in the following.

The reduced or current-value Hamilton function connected to the minimisation problem is obtained by introducing the reduced costate $p=y \mathrm{e}^{\rho t}$ :

$$
H(x, p)=\mathcal{H}\left(t, x, p \mathrm{e}^{-\rho t}\right) \mathrm{e}^{\rho t}=p \psi(x, p)-g(x, \psi(x, p)) .
$$

Note that $H_{p p}$ is the inverse of $g_{\dot{x} \dot{x}}$; hence strict convexity of $g$ with respect to $\dot{x}$ implies strict convexity of $H$ with respect to $p$. As in the following the reduced Hamilton function will be considered mostly, it will cause no confusion to call $p$ the 'costate' instead of 'reduced costate'.

The reduced canonical equations read as

$$
\dot{x}=H_{p}, \quad \dot{p}=\rho p-H_{x} .
$$

Solution trajectories $(x, p)$ of these equations are called (reduced) phase curves. Note that the system (5) has the form of a canonical system if $\rho=0$.

2.3 Situation. The following standing assumptions describe the situation to be considered in the rest of the article. They depend on a nonnegative real constant $\rho_{0}$.

\section{Standing assumption}

1. The equations (5) have for $\rho=\rho_{0}$ two equilibria $e_{1}, e_{2} \in X \times \mathbb{R}^{n}$ that are of saddle type, with stable and unstable manifolds having both dimension $n$. The coordinates of $e_{j}$ are denoted by $\left(x_{j}, p_{j}\right)$.

From this assumption, it follows by the implicit function theorem that there is a constant $\varepsilon_{1}>0$, such that for every $\rho \in\left(\rho_{0}-\varepsilon_{1}, \rho_{0}+\varepsilon_{1}\right)$, there are two saddle equilibria $e_{1}(\rho), e_{2}(\rho)$ whose stable and unstable manifolds have both dimension $n$, and such that $e_{j}\left(\rho_{0}\right)=e_{j}, j=1,2$. In the following, $e_{j}$ will denote the family $\rho \mapsto e_{j}(\rho)$.

\section{Standing assumption}

2. For $\rho=\rho_{0}$, the stable manifold $W_{j}^{s}$ of $e_{j}\left(\rho_{0}\right), j=1,2$, can locally around $e_{j}\left(\rho_{0}\right)$ be represented as the graph of a smooth function. That is, if $E_{j}^{s}$ denotes the tangent space $\mathrm{T} W_{j}^{s}$ to $W_{j}^{s}$ at $e_{j}\left(\rho_{0}\right)$, the derivative $\mathrm{d} \pi: E_{j}^{s} \rightarrow \mathrm{T} \mathcal{X} \cong \mathbb{R}^{n}$ of the projection $\pi$, restricted to $E_{j}^{s}$, is invertible.

Using continuity, there is a positive constant $\varepsilon_{2} \leq \varepsilon_{1}$, such that for each $\rho \in\left(\rho_{0}-\varepsilon_{2}, \rho_{0}+\right.$ $\left.\varepsilon_{2}\right)$, the projection from the tangent space $E_{j}^{s}(\rho)$ to the stable manifold $W_{j}^{s}$ at $e_{j}(\rho)$ is invertible. Then the stable manifold $W_{j}^{s}$ of $e_{j}, j=1,2$, can locally around $e_{j}$ be represented as the graph of a smooth function. That is, for every $\rho \in\left(\rho_{0}-\varepsilon_{2}, \rho_{0}+\varepsilon_{2}\right)$ there is an open set $U_{j} \in X$ that has the following properties: it contains $x_{j}$, and the intersection of $W_{j}^{s}$ with $U_{j} \times \mathbb{R}^{n}$ is equal to the graph of a smooth function $x \mapsto w_{j}^{s}(x, \rho)$ that takes values in $\mathbb{R}^{n}$. By taking unions of sets with these properties, it can be obtained that $U_{j}$ is maximal, 
that is, there is no larger open set in $X$ with the same properties. Sometimes, the set $U_{j}$ is denoted by $U_{j}(\rho)$, in order to emphasise the dependence on $\rho$. Note however that the maximal sets $U_{j}$ need not be unique (think of a stable manifold forming a pleat).

\section{Standing assumption}

3. There is a positive constant $\varepsilon_{3} \leq \varepsilon_{2}$, such that for all $\rho \in\left(\rho_{0}-\varepsilon_{3}, \rho_{0}+\varepsilon_{3}\right) \cap(0, \infty)$, and for every $\xi \in \mathcal{X}$, a minimiser of $J$ exists in $\mathcal{D}_{\xi}$; moreover, its phase trajectory is contained in either the graph of $w_{1}^{s}$ or in that of $w_{2}^{s}$.

Whereas the previous two assumptions were local, the present assumption is of a global nature. The standing assumptions reflect common situations; an investigation into the precise conditions under which the last assumption holds is however matter for future research.

2.4 The optimal vector field. If $x$ is a minimiser, then under the assumptions the phase trajectory of $x$ is contained in the graph of $w_{j}^{s}$, and the first of the reduced canonical equations (5) can be written as

$$
\dot{x}=H\left(x, w_{j}^{s}(x)\right) \stackrel{\text { def }}{=} \varphi(x) ;
$$

that is, optimal trajectories are solution curves of the optimal vector field $\varphi$. This vector field is not well-defined at $\xi \in X$ if $\xi$ is initial point to distinct optimal solution trajectories. Such a point $\xi$ is called an indifference threshold.

The existence of this optimal vector field illustrates the optimality principle: if the trajectory $x$ is a minimiser of $J$ in $\mathcal{D}_{\xi}$, then for every $\tau>0$, the trajectory $\hat{x}_{\tau}$ given as $\hat{x}_{\tau}(t)=x(\tau+t)$ is a minimiser of $J$ in $\mathcal{D}_{x(\tau)}$. Note in particular that since $H$ and $w_{j}^{s}$ are smooth, the minimiser $x$ will be smooth as well, since it is a solution trajectory of the smooth vector field $\varphi$.

Let $B_{j} \subset \mathcal{X}$ be the largest open set such that for every $\xi \in B_{j}$ there is a unique minimiser of $J$ in $\mathcal{D}_{\xi}$ whose phase trajectory tends to $e_{j}$ as time tends to infinity. The set $U_{j}$ can be chosen such that $B_{j} \subset U_{j}$. A point $\xi$ in the boundary of $B_{j}$ is, by definition of $B_{j}$, an indifference point, for which there are two minimisers of $J$ in $\mathcal{D}_{\xi}$. These minimisers may tend to the same or to different long-run steady states.

In general the sets $B_{j}$ and $U_{j}$ are not equal: the boundary points of $B_{j}$ are indifference points, whereas the boundary points of $U_{j}$ are those points for which the stable manifold $W_{j}^{s}$ has a "vertical" tangent, or, put differently, for which the projection $\pi: W_{j}^{s} \rightarrow X$ is singular.

On $B_{j}$, the optimal vector field $\varphi$ is uniquely defined. The projection $x_{j}$ of $e_{j}$ onto the state space is the unique asymptotically stable equilibrium of $\varphi$ in $B_{j}$. Consequently, the set $B_{j}$ is simply connected: every closed curve in $B_{j}$ is homotopic to a point, since it is mapped to $e_{j}$ by a homotopy that is given by the time forward flow of the optimal vector field.

In the following, the arguments of the various functions will be suppressed whenever this is convenient, in order not to burden the notation too much. 
2.5 Indifference thresholds. From equation (3), it follows that along a solution trajectory $(x, y)$ of the canonical equations (4)

$$
\frac{\mathrm{d}}{\mathrm{d} t} \mathcal{H}=\mathcal{H}_{t}=\rho g \mathrm{e}^{-\rho t} .
$$

This holds for any phase curve, not just for those corresponding to minimisers. In particular, if

$$
\lim _{t \rightarrow \infty} \mathcal{H}(t, x(t), y(t))=\lim _{t \rightarrow \infty} H(x(t), p(t)) \mathrm{e}^{-\rho t}=0,
$$

equation (7) implies for a phase curve $(x, y)$ that

$$
\mathcal{H}(0, x(0), y(0))=H(x(0), p(0))=-\rho \int_{0}^{\infty} g(x, \dot{x}) \mathrm{e}^{-\rho t} \mathrm{~d} t .
$$

Fix $\rho_{0}>0$, assume that the standing assumptions hold for $\rho_{0}$, and take $\rho=\rho_{0}$. Define the function $V_{j}: U_{j} \rightarrow \mathbb{R}$ for $\xi \in U_{j}$ as

$$
V_{j}(\xi)=-\frac{1}{\rho} H\left(\xi, w_{j}^{s}(\xi)\right)
$$

Note that if a minimiser of $J$ in $\mathcal{D}_{\xi}$ lies in the stable manifold of $e_{j}$, then $V_{j}(\xi)$ will be equal to the value of the value function $V(\xi)$. The function $V_{j}$ is clearly smooth everywhere on $U_{j}$.

LEMMA 1. If $t \mapsto(x(t), p(t))$ is a reduced phase curve in the stable manifold $W_{j}^{s}$ of $e_{j}$, then

$$
p(t)=-\frac{\partial V_{j}}{\partial x}(x(t))
$$

for all $t \geq 0$.

Though the relation is well-known (see Gelfand and Fomin [8], p. 89-90), care is taken in the proof to show that it only depends on properties of the (reduced) canonical system.

\section{Proof}

Take $\xi \in U_{j}$ and $h \in \mathbb{R}^{n}$. Let $x$ be the projection to state space of the phase curve starting at $\left(\xi, w_{j}^{s}(\xi)\right)$. Since $U_{j}$ is open, the state $\xi+\varepsilon h$ lies also in $U_{j}$ if $\varepsilon \in \mathbb{R}$ is sufficiently close to 0 . Denote by $x_{\varepsilon}(t)=x(t)+\varepsilon \eta(t, \varepsilon)$ the corresponding projection of the phase curve starting at $\left(\xi+\varepsilon h, w_{j}^{s}(\xi+\varepsilon h)\right)$. Since the phase curves corresponding to $x$ and $x_{\varepsilon}$ are both in the stable manifold of $e_{j}$, it follows that $\eta(t, \varepsilon) \rightarrow 0$, uniformly in $t$, as $\varepsilon \rightarrow 0$. Consequently

$$
\begin{aligned}
\frac{V_{j}(\xi+\varepsilon h)-V_{j}(\xi)}{\varepsilon} & =\int_{0}^{\infty}\left(g_{x}(x, \dot{x}) \eta+g_{\dot{x}}(x, \dot{x}) \dot{\eta}\right) \mathrm{e}^{-\rho t} \mathrm{~d} t+\mathrm{o}(\varepsilon) \\
& =\left.g_{\dot{x}} \mathrm{e}^{-\rho t} \eta\right|_{0} ^{\infty}+\int_{0}^{\infty}\left[g_{x} \mathrm{e}^{-\rho t}-\frac{\mathrm{d}}{\mathrm{d} t}\left(g_{\dot{x}} \mathrm{e}^{-\rho t}\right)\right] \eta \mathrm{d} t+\mathrm{o}(\varepsilon)
\end{aligned}
$$


in the last equality integration by parts has been used. Remark that the factor in square brackets is equal to $-\left(\dot{y}+\mathcal{H}_{x}\right)$, and that it is therefore identically zero. Moreover, the limit $\lim _{t \rightarrow \infty} \eta(t, \varepsilon)=0$, uniformly in $\varepsilon$. It follows that

$$
\frac{\partial V_{j}}{\partial x}(\xi) h=-g_{\dot{x}} \eta(0)=-g_{\dot{x}} h,
$$

for all $h \in \mathbb{R}^{n}$. For the reduced phase trajectory $(x, p)$, we conclude that $\partial V_{j} / \partial x+g_{\dot{x}}=0$, or, equivalently, that

$$
p(t)=-\frac{\partial V_{j}}{\partial x}(x(t))
$$

for all $t$.

Note that equation (10) implies that $w_{j}^{s}(\xi)=-\frac{\partial V_{j}}{\partial x}(\xi)$, for all $\xi \in U_{j}$. Under the standing assumptions, the value function satisfies

$$
V(\xi)=\min \left\{V_{1}(\xi), V_{2}(\xi)\right\}
$$

The state $\xi$ is an indifference threshold if $V_{1}(\xi)=V_{2}(\xi)$.

THEOREM 1. Let the standing assumptions be satisfied for some $\rho_{0} \geq 0$. Then for $\rho=\rho_{0}$, the set of indifference thresholds in $U_{1} \cap U_{2}$ is locally a smooth manifold.

\section{Proof}

Assume that $\xi \in U_{1} \cap U_{2}$ is an indifference state; then

$$
\frac{\partial V_{1}}{\partial x}(\xi)-\frac{\partial V_{2}}{\partial x}(\xi)=-w_{1}^{s}(\xi)+w_{2}^{s}(\xi) \neq 0 .
$$

The inequality holds, since otherwise the point $(\xi, \eta)$ with $\eta=w_{1}^{s}(\xi)=w_{2}^{s}(\xi)$ would be contained in the stable manifold of both equilibria, which is manifestly impossible. It follows from the implicit function theorem that locally around $\xi$, the equation $V_{1}(x)-$ $V_{2}(x)=0$ defines a smooth codimension- 1 submanifold in $X$.

\section{APPROXimating THE VALUE FUnCTION}

If the discount rate $\rho>0$ is small, phase trajectories of the reduced canonical equations (5) in the stable manifold $W_{j}^{s}$ of $e_{j}(\rho)$ are uniformly close to phase trajectories in the stable manifold of $e_{j}(0)$ of the same equations for $\rho=0$. If trajectories are considered which are restricted to a finite time interval, this remark is immediate. But uniformity holds also for the infinite time interval $[0, \infty)$, since both trajectories converge to the saddle equilibrium, and close to the saddle equilibrium, the convergence speed is determined by the stable eigenvalues. 
3.1 The main approximation. In this subsection, the value function is approximated, for small values of $\rho>0$, in terms of trajectories of the reduced canonical equations for $\rho=0$; put differently, the equations with $\rho>0$ are viewed as perturbations of the equations with $\rho=0$. Therefore, in the following expressions the dependence on $\rho$ is indicated explicitly.

For every $\rho \geq 0$, consider the phase trajectory $\gamma_{\rho}=\left(x_{\rho}, p_{\rho}\right)$ in the graph of $w_{j}^{s}$ that starts at the phase point $\left(\xi, w_{j}^{s}(\xi, \rho)\right)$ and converges to $e_{j}(\rho)$ as $t \rightarrow \infty$. Along this trajectory, let $v(t)=V\left(x_{\rho}(t), \rho\right)$. Then the following identity holds true:

$$
\frac{\mathrm{d} v}{\mathrm{~d} t}=V_{x} \dot{x}_{\rho}=-p_{\rho} \dot{x}_{\rho} .
$$

In the last equality equation (10) has been used. By integration it follows that (recall that $\left.e_{j}=\left(x_{j}, p_{j}\right)\right)$ :

$$
V\left(x_{j}, \rho\right)-V(\xi, \rho)=-\int_{0}^{\infty} p_{\rho} \dot{x}_{\rho} \mathrm{d} t
$$

or equivalently, using (9), that

$$
V(\xi, \rho)=-\frac{1}{\rho} H\left(e_{j}(\rho)\right)+\int_{\gamma_{\rho}} p \mathrm{~d} x
$$

Note that the integral in equation (12) is a line integral: the differential form $p \mathrm{~d} x=$ $\sum_{i=1}^{n} p_{i} \mathrm{~d} x_{i}$ is integrated over the curve $\gamma_{\rho}$. As this curve is contained in the stable manifold $W_{j}^{s}$, along it the identity $p_{\rho}=w_{j}^{s}\left(x_{\rho}, \rho\right)$ holds true.

THEOREM 2. Let the standing assumptions be satisfied for $\rho_{0}=0$. Let $\rho \mapsto \xi_{\rho} \in$ $U_{j}(\rho)$ be a smooth curve, and let $\gamma_{\rho}$ be the phase trajectory of the reduced canonical equations with initial value $\left(\xi_{\rho}, w_{j}^{s}\left(\xi_{\rho}, \rho\right)\right)$. Then there exists a constant $\bar{\rho}>0$ and a smooth function $\beta(\xi, \rho)$, such that for $\rho \in(0, \bar{\rho})$, the function $V_{j}$ satisfies

$$
V_{j}\left(\xi_{\rho}, \rho\right)=-\frac{1}{\rho} H\left(e_{j}(0)\right)+\int_{\gamma_{0}} p \mathrm{~d} x+\rho \beta\left(\xi_{0}, \rho\right) .
$$

\section{Proof}

The strategy of the proof is to show that the two terms on the right hand side of equation (12) can be approximated up to order $\mathrm{O}(\rho)$ by the corresponding terms in equation (13).

Denote by $f(x, p, \rho)$ the reduced canonical vector field $\left(H_{p}, \rho p-H_{x}\right)$, and recall that $e_{j}(\rho)=\left(x_{j}, p_{j}\right)$ solves $f\left(x_{j}, p_{j}, \rho\right)=0$. Moreover, the assumption that $e_{j}$ is of saddle type implies in particular that the Jacobian matrix $D_{(x, p)} f$, evaluated at $\left(x_{j}, p_{j}, \rho\right)$, is invertible. By the implicit function theorem, it follows that $e_{j}$ depends differentiably on $\rho$. 
Since $e_{j}(0)$ solves the equations $H_{p}=H_{x}=0$, Taylor's theorem implies that

$$
H\left(e_{j}(\rho)\right)=H\left(e_{j}(0)+\mathrm{O}(\rho)\right)=H\left(e_{j}(0)\right)+\mathrm{O}\left(\rho^{2}\right) .
$$

This takes care of the first term in (12).

Split the integral in the second term in (12) into two parts

$$
\int_{\gamma_{\rho}} p \mathrm{~d} x=\int_{0}^{T} p_{\rho} \dot{x}_{\rho} \mathrm{d} t+\int_{T}^{\infty} p_{\rho} \dot{x}_{\rho} \mathrm{d} t
$$

where $T$ will be chosen independently of $\rho$. If it is shown that restricted to $[0, T]$ the maximal distance of $\gamma_{\rho}(t)$ to $\gamma_{0}(t)$ is of order $\mathrm{O}(\rho)$, it follows that the difference between the first integral in (15) and its counterpart for $\rho=0$ is also of order $\mathrm{O}(\rho)$. The second integral has to be treated a bit more carefully, since it is taken over an infinite interval. There, uniformity of estimates with respect to $\rho$ will follow from the fact that the convergence towards $e_{j}$ is controlled by the stable eigenvalue closest to 0 .

After this introduction, consider the 'vertical' vector field $F(x, p, \rho)=\left(H_{p}, \rho p-H_{x}, 0\right)$ on $X \times \mathbb{R}^{n} \times[0, \bar{\rho}]$; the use of vertical vector fields is a technical device by which a family of vector fields can be treated as a single vector field on the product of phase space $\left(X \times \mathbb{R}^{n}\right)$ and parameter space $([0, \bar{\rho}])$. Solution curves $(x(t), p(t), \rho(t))$ of the vertical vector field satisfy the equations

$$
\dot{x}=H_{p}, \quad \dot{p}=\rho p-H_{x}, \quad \dot{\rho}=0 .
$$

Note that since $e_{j}(\rho)$ is an equilibrium for every $\rho$, the curve $c: \rho \rightarrow\left(e_{j}(\rho), \rho\right)$ is invariant under the flow of $F$.

The linearisation $D_{(x, p, \rho)} F$ of the vector field at the equilibrium $e_{j}$ has $n$ stable and $n$ unstable eigenvalues, as well as an eigenvalue 0 . By the fundamental theorem on normally hyperbolic invariant manifolds (see Hirsch, Pugh and Shub [11]), there is a smooth stable manifold and a smooth unstable manifold through the curve $c$ of equilibria, fibered into smooth stable manifolds and smooth unstable manifolds tangent to the stable manifolds of $e_{j}(\rho)$.

In other words, stable and unstable manifolds of the family of equations (5), which are parametrised by $\rho$, depend smoothly on $\rho$. Consequently, the distance of the initial points $\left(\xi_{0}, w_{j}^{s}\left(\xi_{0}, 0\right)\right)$ and $\left(\xi_{\rho}, w_{j}^{s}\left(\xi_{\rho}, \rho\right)\right)$ of the curves $\gamma_{0}$ and $\gamma_{\rho}$ is also bounded by $c_{1} \rho$. Moreover, since $\rho \mapsto e_{j}(\rho)$ is differentiable, there are constants $c_{2}, \rho_{2}>0$ such that $\left|e_{j}(\rho)-e_{j}(0)\right| \leq c_{2} \rho$ for all $0 \leq \rho \leq \rho_{2}$.

Take $\bar{\rho}=\min \left\{\rho_{1}, \rho_{2}\right\}$. For every $\rho \in[0, \bar{\rho}]$, there is a $T_{\rho}>0$ such that if $t>T_{\rho}$, then

$$
\left|\gamma_{\rho}(t)-e_{j}(\rho)\right|<c_{3} \rho
$$

moreover, $T_{\rho}$ depends continuously on $\rho$. Let $T=\max _{\rho \in[0, \bar{\rho}]} T_{\rho}$. Then it follows by the continuous dependence on initial conditions of solutions of differential equations that there 
is a constant $c_{4}>0$ such that for all $0 \leq \rho \leq \bar{\rho}$ :

$$
\max _{t \in[0, T]}\left|\gamma_{\rho}(t)-\gamma_{0}(t)\right|<c_{4} \rho
$$

This implies that

$$
\left|\int_{0}^{T} p_{\rho} \dot{x}_{\rho} \mathrm{d} t-\int_{0}^{T} p_{0} \dot{x}_{0} \mathrm{~d} t\right|<c_{5} \rho
$$

As indicated above, this takes care of the first integral on the right hand side of (15).

Turn now to the second integral in (15). Restricted to the stable manifold $W_{j}^{s}$, the linearisation of the vector field $\left(H_{p}, \rho x-H_{x}\right)$ at the equilibrium $e_{j}(\rho)$ has $n$ stable eigenvalues $\lambda_{i}^{S}(\rho)$, which depend continuously on $\rho$. Take $\alpha>0$ such that

$$
\max _{\rho \in[0, \bar{\rho}]} \operatorname{Re} \lambda_{i}^{s}(\rho)<-\alpha, \quad i=1, \cdots, n .
$$

It is a standard result from the stability theory of differential equations (see e.g. [4], p. 92, or [1], p. 25), that there is a constant $c_{6}>0$, which is independent of $\rho$, such that

$$
\left|\gamma_{\rho}(t)-e_{j}\right|<c_{6} \mathrm{e}^{-\alpha t}\left|\gamma_{\rho}(T)\right|
$$

for all $t>T$. Since $\dot{x}=H_{p}$ and $H_{p}\left(e_{j}\right)=0$, it follows for $t>T$ and $0 \leq \rho \leq \bar{\rho}$ that

$$
\left|\dot{x}_{\rho}(t)\right|=\left|H_{p}\left(\gamma_{\rho}(t)\right)-H_{p}\left(e_{j}\right)\right|<c_{7}\left|\gamma_{\rho}(t)-e_{j}\right|<c_{8} e^{-\alpha t} \rho .
$$

In the first inequality, the mean value estimate has been used; in the second, inequalities (17) and (19).

Using the estimate (20) and again inequality (17), the final result

$$
\left|\int_{T}^{\infty} p \dot{x} \mathrm{~d} t\right| \leq c_{9} \rho
$$

is obtained, with $c_{9}$ independent of $\rho$. In an analogous manner, invoking uniform convergence again, it follows that the integral $\int_{T}^{\infty} p \dot{x} \mathrm{~d} t$ depends smoothly on $\xi$ and $\rho$. Combining inequalities (14), (18) and (21) yields the result.

3.2 Consequences. Theorem 2 can be applied in various ways. For instance, if the state space is one-dimensional, the reduced Hamilton function $H$ is a first integral of the equations, and the phase curves are contained in the level curves of $H$. If the level sets can be determined analytically, the approximation to $V_{j}$ in (13) can be determined by integration.

To develop a more general application of the result, assume that the optimalisation problem depends on a second parameter $\lambda$. Assume that there is a value $\lambda_{0}$ which is such 
that for $\rho=0$, the value of the reduced Hamilton function at the two equilibria is equal. If for fixed $C>0$ attention is restricted to the cone

$$
K=\left\{(\lambda, \rho):\left|\lambda-\lambda_{0}\right| \leq C \rho, \rho \geq 0\right\}
$$

in the space of parameters, then equation (13) can be replaced by

$$
V_{j}\left(\xi_{\rho}, \lambda, \rho\right)=-\frac{1}{\rho} h_{j}\left(\lambda_{0}\right)+\frac{\partial h_{j}}{\partial \lambda}\left(\lambda_{0}\right) \frac{\lambda-\lambda_{0}}{\rho}+\int_{\gamma_{j}} p \mathrm{~d} x+\rho \beta\left(\xi_{0}, \frac{\lambda-\lambda_{0}}{\rho}, \rho\right),
$$

where $h_{j}(\lambda)=H\left(e_{j}(\lambda), \lambda\right)$, where $\xi_{\rho}$ is a curve of initial conditions for which $V_{j}$ is defined, and where $\gamma_{j}$ is the phase curve of the reduced canonical equations starting at $\left(\xi_{0}, w_{j}^{s}\left(\xi_{0}, \lambda_{0}, 0\right)\right)$.

At every point $\xi_{\rho}$ for which both $V_{1}$ and $V_{2}$ are defined, their difference is given as $\Delta\left(\xi_{\rho}\right)=V_{2}\left(\xi_{\rho}\right)-V_{1}\left(\xi_{\rho}\right)$. Combining the approximations yields

$$
\Delta\left(\xi_{\rho}\right)=\left(b_{2}-b_{1}\right) \frac{\lambda-\lambda_{0}}{\rho}+\delta\left(\xi_{0}\right)+\mathrm{O}(\rho)
$$

where $\delta\left(\xi_{0}\right)$ is equal to $\int_{\gamma_{2}} p \mathrm{~d} x-\int_{\gamma_{1}} p \mathrm{~d} x$. It follows that the indifference manifolds $\Delta=0$ are, for varying $\lambda$, up to order $\mathrm{O}(\rho)$ equal to the level sets of the function $\delta$. In particular, for small discount rates indifference manifolds for different values of $\lambda$ will not intersect each other.

If the phase curve of the reduced Hamilton equations for $\rho=0$ cannot be determined analytically, equation (13) can be used as the basis of a numerical scheme. Discretise the problem by laying a grid over phase space; for every gridpoint, the value of the integral $\int_{\gamma_{j}} p \mathrm{~d} x$ can be determined numerically (this is a nontrivial numerical problem if the dimension of the state space is larger than 1). Then equation (13) gives an approximation to the value function for a whole range of values of $\rho$ close to 0 .

In particular, for every grid point $X_{k}$, an approximation $V_{j k}$ of $V_{j}\left(X_{k}\right)$ is given by

$$
V_{j k}=\frac{a}{\rho}+b_{j} \frac{\lambda-\lambda_{0}}{\rho}+c_{j k}
$$

where $a=h_{1}\left(\lambda_{0}\right)=h_{2}\left(\lambda_{0}\right), b_{j}=\frac{\partial h_{j}}{\partial \lambda}\left(\lambda_{0}\right)$, and $c_{j k}$ is the value of the integral $\int_{\gamma_{j}} p \mathrm{~d} x$, evaluated for $\rho=0$ over the phase trajectory starting at $\left(X_{k}, w_{j}^{s}\left(X_{k}, \lambda_{0}, 0\right)\right)$ that tends to $e_{j}$ as $t \rightarrow \infty$. For every point $X_{k}$ we can now approximate $\Delta\left(X_{k}\right)$, and determine numerical approximations to the indifference manifolds by interpolation. Remark that this procedure does not depend on the dimension of the state space; in particular, approximate indifference manifolds in two- and three-dimensional manifolds can be computed in this way.

The moral of the approximation method is that only one integration is necessary to obtain information on the system for a range of parameters. 


\section{EXAMPLE}

In this section, an example is considered for which all approximations can be calculated explicitly; by calculating the 'true' values as well, the approximations are seen to be quite acceptable.

Let $X=\mathbb{R}$ and consider the following functional

$$
J=\int_{0}^{\infty}\left(\frac{\dot{x}^{2}}{2}-\frac{x^{2}}{2}-\frac{2}{3} \lambda x^{3}+\frac{x^{4}}{4}\right) \mathrm{e}^{-\rho t} \mathrm{~d} t .
$$

Note that $p=\dot{x}$ and that

$$
H(x, p, \lambda)=\frac{p^{2}}{2}+\frac{x^{2}}{2}+\frac{2}{3} \lambda x^{3}-\frac{x^{4}}{4} .
$$

The reduced canonical equations read as

$$
\dot{x}=p, \quad \dot{p}=\rho p-x-2 \lambda x^{2}+x^{3} .
$$

Equilibria are given by $(\xi, 0)$, where $\xi \in\left\{\lambda-\sqrt{1+\lambda^{2}}, 0, \lambda+\sqrt{1+\lambda^{2}}\right\}$. The second derivative of $H$ at $(\xi, 0)$ reads as

$$
D^{2} H(\xi, 0)=\left(\begin{array}{cc}
0 & 1 \\
3 \xi^{2}-4 \lambda \xi-1 & \rho
\end{array}\right) .
$$

For $\xi=0$, both the determinant and the trace of $D^{2} H(\xi, 0)$ are positive; hence $(\xi, 0)$ is a source. For the other two values of $\xi$, the determinant is negative; these points are saddles. We set $x_{1}=\lambda-\sqrt{1+\lambda^{2}}, x_{2}=\lambda+\sqrt{1+\lambda^{2}}$, and $e_{j}=\left(x_{j}, 0\right)$ for $j=1,2$. Note that the equilibria are independent of $\rho$ in this example.

The values of the Hamiltonian at the equilibria are denoted by $h_{j}(\lambda)=H\left(e_{j}(\lambda), \lambda\right)$; they are given as

$$
h_{1}(\lambda)=\frac{1}{4}+\lambda^{2}+\frac{2}{3} \lambda^{4}-\frac{2}{3} \lambda\left(1+\lambda^{2}\right) \sqrt{1+\lambda^{2}}
$$

and

$$
h_{2}(\lambda)=\frac{1}{4}+\lambda^{2}+\frac{2}{3} \lambda^{4}+\frac{2}{3} \lambda\left(1+\lambda^{2}\right) \sqrt{1+\lambda^{2}} .
$$

The stable manifold of $e_{j}$ is, for $\rho=0$, contained in the level set $H=h_{j}$; this yields

$$
\begin{aligned}
& w_{1}^{s}(x, \lambda, 0)=-\sqrt{2\left(h_{1}-\frac{x^{2}}{2}-2 \lambda \frac{x^{3}}{3}+\frac{x^{4}}{4}\right)}, \\
& w_{2}^{s}(x, \lambda, 0)=\sqrt{2\left(h_{2}-\frac{x^{2}}{2}-2 \lambda \frac{x^{3}}{3}+\frac{x^{4}}{4}\right)} .
\end{aligned}
$$


Note that $w_{j}^{s}$ is a smooth function for all $x$ such that the expression under the square root is positive; hence

$$
U_{j}=\left\{x \in X: \frac{x^{2}}{2}+2 \lambda \frac{x^{3}}{3}-\frac{x^{4}}{4}<h_{j}\right\} .
$$

For an indifference point $\xi$ we have that $V_{1}(\xi)=V_{2}(\xi)$. Using equation (13) and taking into account the direction of integration, the following equation is obtained for an indifference point $\xi$ :

$$
0=-\frac{1}{\rho}\left(h_{2}(\lambda)-h_{1}(\lambda)\right)+\int_{\xi}^{x_{2}} w_{2}^{s}(x, \lambda, 0) \mathrm{d} x+\int_{x_{1}}^{\xi} w_{1}^{s}(x, \lambda, 0) \mathrm{d} x+\mathrm{O}(\rho) .
$$

The difference $h_{2}-h_{1}$ is equal to

$$
h_{2}(\lambda)-h_{1}(\lambda)=\frac{4}{3} \lambda\left(1+\lambda^{2}\right) \sqrt{1+\lambda^{2}} .
$$

From equation (22) we want to solve $\xi$ as a function of $\lambda$; however, it turns out that it is more convenient to express $\lambda$ as a function of $\xi$ by putting $\lambda=\rho \mu(\xi, \rho)$. Substitution in the equation, and letting $\rho$ tend to 0 , leads then to

$$
\frac{4}{3} \mu(\xi, 0)=\int_{\xi}^{x_{2}(0)} w_{2}^{s}(x, 0,0) \mathrm{d} x+\int_{x_{1}(0)}^{\xi} w_{1}^{s}(x, 0,0) \mathrm{d} x .
$$

Since $h_{1}(0)=h_{2}(0)=\frac{1}{4}$, the functions $w_{1,2}^{s}$ are for $(\lambda, \rho)=(0,0)$ given by

$$
w_{1}^{s}(x, 0,0)=-\frac{1}{2} \sqrt{2}\left(1-x^{2}\right), \quad w_{2}^{s}(x, 0,0)=\frac{1}{2} \sqrt{2}\left(1-x^{2}\right) .
$$

The integrals in (23) can be evaluated explicitly; they yield

$$
\mu(\xi, 0)=\frac{3}{8} \sqrt{2}\left(\int_{\xi}^{1}\left(1-x^{2}\right) \mathrm{d} x-\int_{-1}^{\xi}\left(1-x^{2}\right) \mathrm{d} x\right)=\frac{\sqrt{2}}{4}\left(\xi^{3}-3 \xi\right) .
$$

Inverting this relation gives a function $\xi=\xi(\mu)$, defined on the interval $\left(-\frac{\sqrt{2}}{2}, \frac{\sqrt{2}}{2}\right)$, which indicates for every value of $\mu=\lambda / \rho$ the approximate position of the indifference point. Using this function, and the positions of the equilibria obtained above, a 'bifurcation diagram' of the system is drawn in figure 1.

In the same way, an approximation to the value function can be found, again for $(\lambda, \rho)$ in some cone $\{|\lambda|<C \rho, \rho \geq 0\}$ of the parameter space. In the present example, equation (13) reads for $j=1$ as

$$
\begin{aligned}
V_{1}(\xi, \lambda, \rho) & =-\frac{1}{\rho}\left(\frac{1}{4}-\frac{2}{3} \lambda\right)+\int_{-1}^{\xi}\left(1-x^{2}\right) \mathrm{d} x+\mathrm{O}(\rho) \\
& =-\frac{1}{4 \rho}+\frac{2}{3} \frac{\lambda}{\rho}+\frac{2}{3}+\xi-\xi^{3} / 3+\mathrm{O}(\rho) .
\end{aligned}
$$




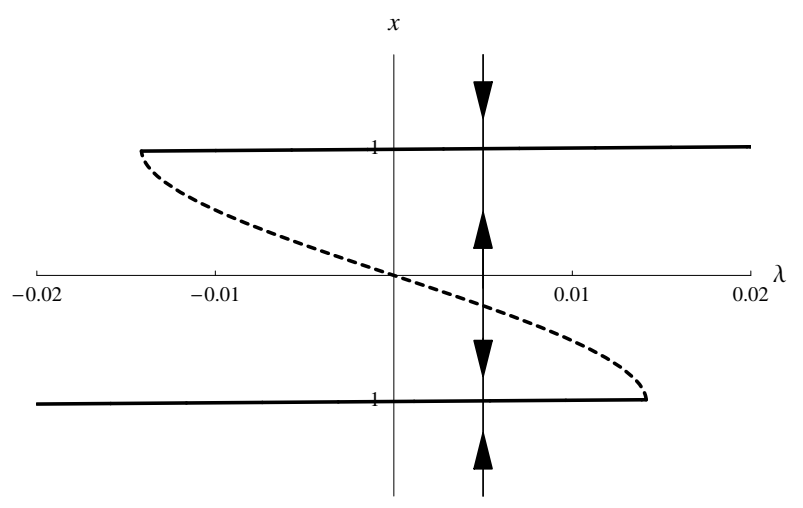

Figure 1: Bifurcation diagram in the $(\lambda, x)$-space for $\rho=0.02$. Shown is for every $\lambda$ the approximated location $\xi(\lambda / \rho)$ of the indifference point (broken line) and the locations of the equilibria of the optimal vector fi eld (solid lines). Also indicated is the direction of the optimal vector fi eld. At $\lambda \approx-0.014$, an indifference threshold and an optimal stable equilibrium are created in an threshold-node bifurcation. The threshold disappears in another such bifurcation for $\lambda \approx 0.014$.

For $V_{2}$, we obtain

$$
\begin{aligned}
V_{2}(\xi, \lambda, \rho) & =-\frac{1}{\rho}\left(\frac{1}{4}+\frac{2}{3} \lambda\right)+\int_{\xi}^{1}\left(1-x^{2}\right) \mathrm{d} x+\mathrm{O}(\rho) \\
& =-\frac{1}{4 \rho}-\frac{2}{3} \frac{\lambda}{\rho}+\frac{2}{3}-\xi+\xi^{3} / 3+\mathrm{O}(\rho) .
\end{aligned}
$$

In figure 2 the quality of these approximations is shown for $(\lambda, \rho)=(0.01,0.02)$ and $(0.1,0.2)$, respectively. They are compared to the 'true' values of the functions $V_{j}$, which have been computed by integrating equation (11) along phase curves of the reduced canonical equations, taking as starting point $V_{j}\left(x_{j}, \rho\right)=-H\left(e_{j}(\rho)\right) / \rho$ at the equilibrium $e_{j}$. Note that a fixed constant has been substracted of $V_{1}$ and $V_{2}$.

Figure 3 shows the phase diagrams for $\rho=0.2$ and $\lambda$ ranging from -0.16 to 0.16 . When appropriate, the location of the approximation $\xi(\lambda / \rho)$ to the indifference threshold is given as well. Also given are the functions $V_{1}$ and $V_{2}$; the value function is their minimum. Note that $V_{1}$ and $V_{2}$ are never both defined for all states.

\section{ACKNOWLEDGEMENTS}

Some of the results in the present article have been presented at the Skiba satellite workshop of the Eight Viennese Workshop on Optimal Control, Dynamic Games and Nonlinear Dynamics. The author wishes to thank warmly Christophe Deissenberg and three anonymous referees for comments that have helped to improve the article, as well as the Nether- 

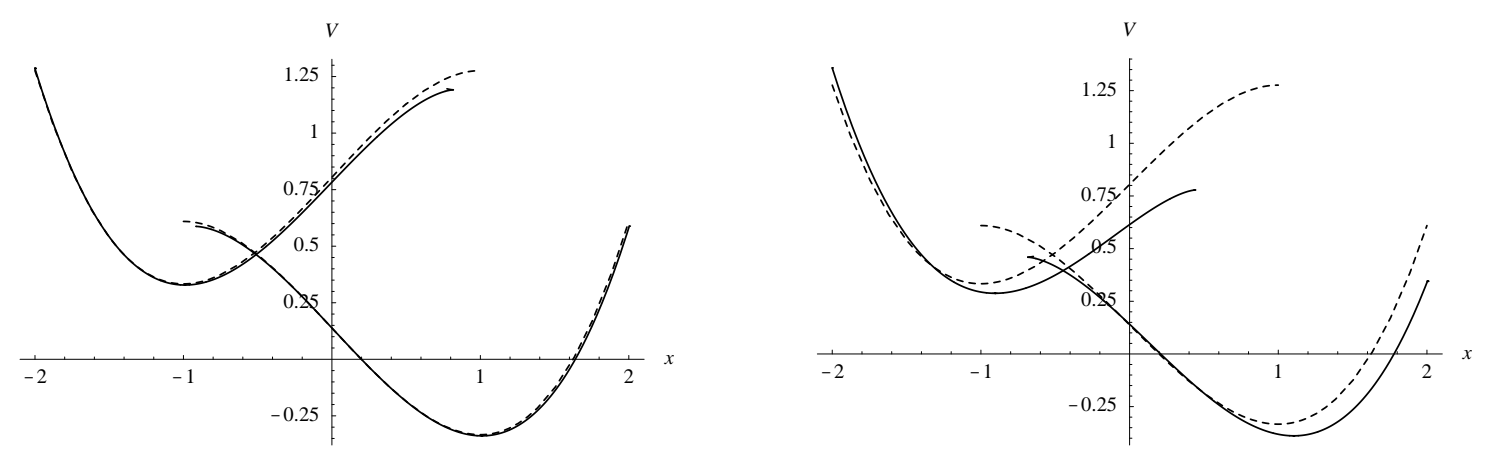

Figure 2: True (drawn) and approximate (broken) values of the functions $V_{1}+1 /(4 \rho)$ and $V_{2}+$ $1 /(4 \rho)$ for $(\lambda, \rho)=(0.01,0.02)$ (left) and $(0.1,0.2)$ (right).

lands Organisation for Science (NWO) for financial support through the CeNDEF Pionier project. 

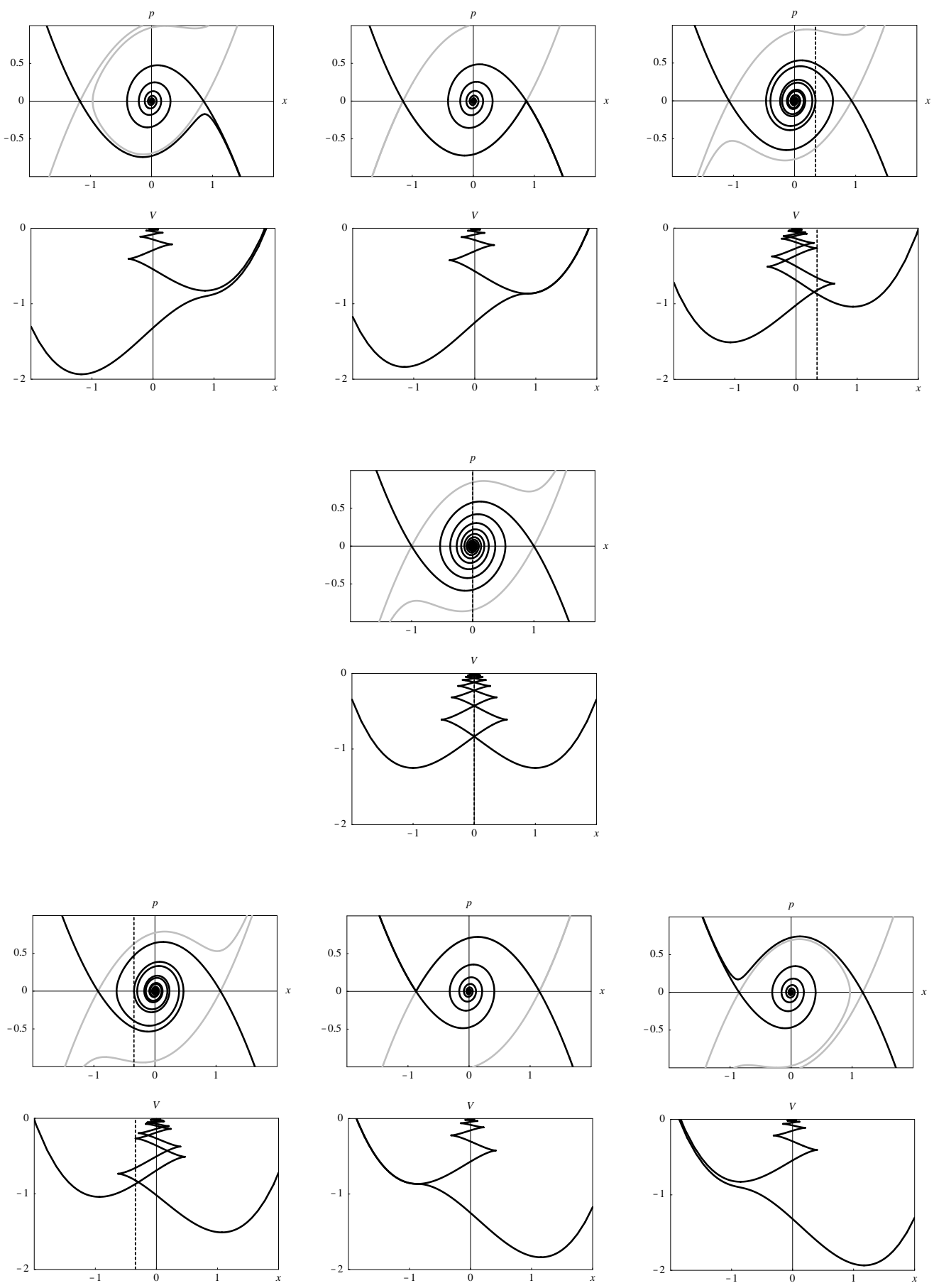

Figure 3: Phase diagrams (odd rows) and value functions (even rows) for $\rho=0.2$ and $\lambda$ taking consecutively the values $-0.16,-(\rho / 2) \sqrt{2},-0.16,-0.07,0,0.07,(\rho / 2) \sqrt{2}$ and 0.16 . The black curves in the phase plots are the stable manifolds, the grey curves are unstable manifolds. The location of the approximation $\xi(\lambda / \rho)$ of the indifference threshold is indicated by a broken line. Note that for $\lambda$ taking one of the bifurcation values, which are approximately equal to $\pm(\rho / 2) \sqrt{2}$, the value function is $C^{1}$ but not $C^{2}$. In between these, the value function is only continuous, for $\lambda$ above $(\rho / 2) \sqrt{2}$ and below $-(\rho / 2) \sqrt{2}$ it is smooth $\left(C^{\infty}\right)$. 


\section{REFERENCES}

[1] D.V. Anosov, S.Kh. Aranson, V.I. Arnol'd, I.U. Bronshtein, V.Z. Grines, and Yu.S. Il'yashenko, Ordinary differential equations and smooth dynamical systems, Springer, Heidelberg, 1988, (Third edition 1997).

[2] W.A. Brock and A.G. Malliaris, Differential equations, stability and chaos in dynamical systems, North-Holland, 1989.

[3] W.A. Brock and D. Starrett, Nonconvexities in ecological management problems, University of Wisconsin, http: / /www.ssc.wisc.edu/econ/archive, 1999.

[4] L. Cesari, Asymptotic behavior and stability problems in ordinary differential equations, Ergebnisse der Mathematik und ihrer Grenzgebiete, vol. 16, Springer, Berlin, 1959.

[5] W.D. Dechert and W.A. Brock, The lake game, Preprint, 2000.

[6] W.D. Dechert and K. Nishimura, A complete characterization of optimal growth paths in an aggregated model with a non-concave production function, Journal of Economic Theory 31 (1983), 332-354.

[7] Ch. Deissenberg, G. Feichtinger, W. Semmler, and F. Wirl, History dependent multiple equilibria and global dynamics in efficient intertemporal optimization models, Economic Complexity: Non-linear Dynamics, Multi-agents Economies, and Learning (W. Barnett, Ch. Deissenberg, and G. Feichtinger, eds.), ISETE, vol. 14, Elsevier, Amsterdam, 2004, pp. 91-122.

[8] I.M. Gelfand and S.V. Fomin, Calculus of variations, Prentice-Hall, Englewood Cliffs, 1963.

[9] L. Grüne and W. Semmler, Using dynamic programming with adaptive grid scheme for optimal control problems in economics, Journal of Economic Dynamics and Control (2004), In Press.

[10] J.L. Haunschmied, P.M. Kort, R.F. Hartl, and G. Feichtinger, A DNS-curve in a twostate capital accumulation model: a numerical analysis, Journal of Economic Dynamics \& Control 27 (2003), 701-716.

[11] M.W. Hirsch, C.C. Pugh, and M. Shub, Invariant manifolds., Lecture Notes in Mathematics, vol. 583, Springer, Heidelberg, 1977.

[12] K.L. Judd, Computational economics and economic theory: substitutes or complements?, Journal of Economic Dynamics \& Control 21 (1997), no. 6, 907-942.

[13] K.-G. Mäler, A. Xepapadeas, and A. de Zeeuw, The economics of shallow lakes, Environmental and Resource Economics (2003), 105-126.

[14] L.S. Pontryagin, V.G. Boltyanskii, R.V. Gamkrelidze, and E.F. Mishchenko, The mathematical theory of optimal processes, Wiley, 1962. 
[15] F. Riesz and B. Sz.-Nagy, Leçons d'analyse fonctionelle, Académie des Sciences de Hongrie, 1952.

[16] A.K. Skiba, Optimal growth with a convex-concave production function, Econometrica 46 (1978), 527-539.

[17] F.O.O. Wagener, Skiba points and heteroclinic bifurcations, with applications to the shallow lake system, Journal of Economic Dynamics and Control 27 (2003), 15331561.

[18] _ Structural analysis of optimal investment for firms with non-concave revenues, CeNDEF Working paper 03-07, 2003. 